\title{
The Rotational Kinematic Formula Viewed from a Tetrad-based Anholonomic Frame
}

\author{
G. Hugh SonG* \\ Department of Physics and Photon Science, Gwangju Institute of Science and Technology, Gwangju 61005, Korea
}

(Received 10 January 2020; revised 14 January 2020; accepted 15 January 2020)

\begin{abstract}
We give a fresh look into the age-old rotational kinematic formula which was originally devised by L. Euler in the eighteenth century. Instead of some verbose explanations for its logical validity, an argument of a covariant differentiation with respect to coordinate time with a tetrad-based relativistic account in an anholonomic frame will be given as a viewpoint that is mathematically sound and self-explanatory. The familiar " $\boldsymbol{\omega}(t) \times \boldsymbol{q}(t)$ " term is replaced by a linear combination of space-frame fields of a tetrad with 'Ricci's connection coefficients' of infinitesimal generators, so as to be expressed by $\sum_{i, j=1}^{3} \underline{\mathfrak{e}}_{(i)} c \underline{\gamma}^{i}{ }_{j 0} \stackrel{\circ}{q}^{j}(t)$ with one subscript index set to zero, ' 0 ', for the time coordinate. This recognition gives a new interpretation of the "time derivative for the space set of rotated axes" as the formal covariant time derivative in this tetrad-based coordinate transformation of the four-dimensional space-time that is curved due to an implicit Galilean transformation.
\end{abstract}

PACS numbers: 02.40.Hw, 04.20.Cv, 45.05.+x, 45.20.-d, 45.20.dc

Keywords: Rotational kinematic formula, Tetrad, Ricci connection coefficients, Christoffel symbols, Ricci rotation coefficients, Euler's equations

DOI: $10.3938 / \mathrm{jkps} .76 .357$

\section{INTRODUCTION}

One of the standard subjects in classical mechanics class is the subject of rotational dynamics of a rigidbody. It starts with an important formula invented in 1765, among so many formulas and equations invented or devised by L. Euler (1707-1783, Swiss), called the rotational kinematic formula [1]. The formula has been written in varied shapes that could have been given by a 3-by-1 matrix form

$$
\epsilon_{t} \stackrel{\circ}{\mathbf{q}} \equiv \mathrm{d}_{t} \stackrel{\mathrm{q}}{\mathbf{q}}+\stackrel{\circ}{\Omega}_{\triangleright}(t) \stackrel{\mathrm{q}}{\mathbf{q}}(t)
$$

with a 3 -by-3 antisymmetric matrix $\stackrel{\Omega}{\triangleright}_{\triangleright}(t)$, which we will relate explicitly to 'Ricci's connection coefficients' ' $\left\{\left\{\gamma_{\lambda \mu \nu}\right\}\right\}$ ' in the present monograph. Also, the shape of the above equation with covariant time differentiation will be promoted for wider use from this monograph.

The foregoing kinematic formula is supposed to be taken in the left-hand side of a certain equation of motion for a point particle at

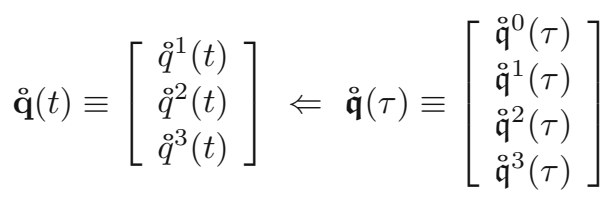

\footnotetext{
*E-mail: hughsong@gist.ac.kr
}

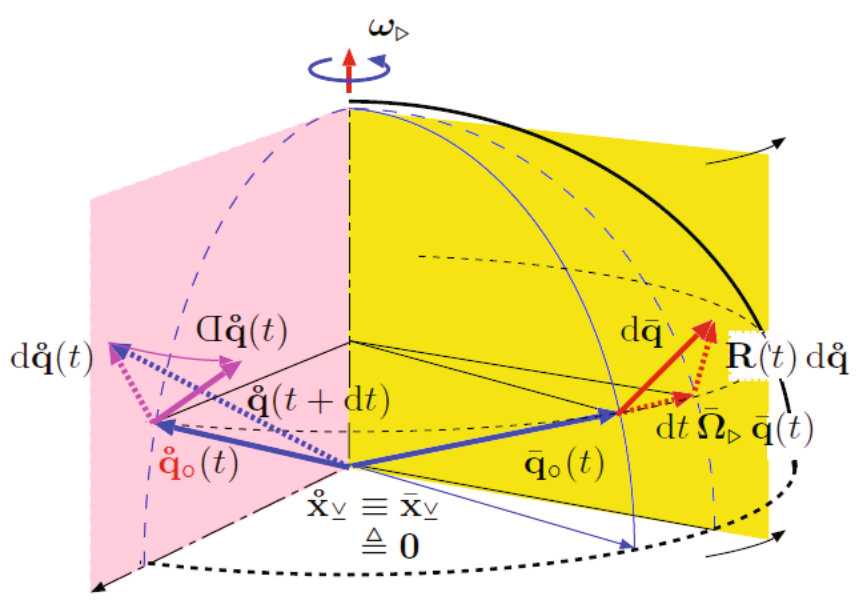

Fig. 1. (Color online) Graphical explanation of $₫ \mathbf{q}(t) \equiv$ $\mathrm{d} \stackrel{\circ}{\mathbf{q}}(t)+\mathrm{d} t \cdot \stackrel{\circ}{\Omega}_{\triangleright}(t) \cdot \stackrel{\circ}{\mathbf{q}}(t)$ by a plot of $\overline{\mathbf{q}}(t)$ on a rotating plane in the bar-accented inertial frame. The total change $\mathrm{d} \overline{\mathbf{q}}(t)$ in the inertial frame is the vector sum of azimuthal component $\mathrm{d} t$. $\overline{\mathbf{\Omega}}_{\triangleright}(t) \overline{\mathbf{q}}(t)$ and its transversal component $\mathbf{R}(t)$ d $\mathbf{q}(t)$, whereas $\circlearrowleft \stackrel{\mathbf{q}}{ }(t)$ is the corresponding vector sum in the loop-accented co-rotating, non-inertial frame.

in the loop-accented non-inertial, orthonormal frame that rotates in time from the bar-accented inertial frame, in which we express the position by $\overline{\mathbf{q}}(t)$. The situation of the rotating non-inertial frame is illustrated schematically in Fig. 1. As is well-known, with the above im- 
portant equation, we are supposed to analyze some of important subjects such as the Coriolis effect and Larmor precession among others.

In Eq. (1), notably distinguished from all other conventional 'take's in literature, listed in Appendix A in the present monograph, we explicitly employ two different differentiation symbols ' $\epsilon_{t}$ ' and ' $\mathrm{d}_{t}$ ' as prefixes to

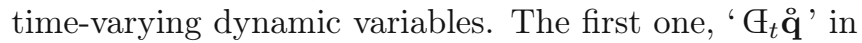
the left-hand side of Eq. (1) will be referred to as 'covariant differentiation',

$$
\Theta_{t} \stackrel{\circ}{\mathbf{q}} \equiv \frac{\mathrm{C}}{\mathrm{d} t} \stackrel{\circ}{\mathbf{q}}(t)
$$

whereas ' $\mathrm{d}_{t} \stackrel{\circ}{\mathbf{q}}$ ' in the right-hand side is the regular differentiation of Newton's quotient limit;

$$
\mathrm{d}_{t} \stackrel{\circ}{\mathbf{q}} \equiv \frac{\mathrm{d}}{\mathrm{d} t} \stackrel{\mathrm{q}}{ }(t) \triangleq \lim _{\epsilon \rightarrow 0} \frac{\stackrel{\mathrm{q}}{(}(t+\epsilon)-\stackrel{\circ}{\mathbf{q}}(t)}{\epsilon}
$$

on the column-matrix expression in Eq. (2). As is briefed in Appendix A, such interpretations on those two differentiation symbols have not been commonly adopted in literature.

In fact, as far as I can find, I have not found any complete account on the tetrad-based interpretation which uses so-called Ricci connection coefficients [2] $\left\{\left\{\gamma_{\mu \nu}^{\lambda}\right\}\right\}$, also known as "Ricci rotation coefficients" as well, instead of Christoffel symbols $\left\{\left\{\Gamma_{\mu \nu}^{\lambda}\right\}\right\}$. That is indeed the issue that I wish to settle by recognizing the true meaning of the time derivative in the so-called "space set of rotated axes". I promote the new interpretation as a new standard presentation of the subject, although such a new presentation employs some slightly-advanced mathematics of differential geometry in the subject of a tetrad of the simple kind with the anholonomic frame carrying a set of four orthonormal frame fields.

Before getting into some mathematics of the subject, one must be acquainted with the historical development on the use of Christoffel symbols and Ricci connection coefficients on Riemannian manifolds in the nineteenth century with no notion of the time variable as one of the coordinates of the geometry. A short chronology of those symbols is presented in Appendix B.

Incidentally, the argument of "rotation coefficients" was once presented in a conference proceeding [3] in 1925. However, the key formula given for Levy's evaluation of Ricci's coefficients of rotation does not appear to have been completed. A good appreciation of the new viewpoint of a tetrad in the present monograph can be achieved only after considering a comparable Galilean transformation into a holonomic frame, non-orthonormal, in parallel to the desired anholonomic frame of a tetrad of four orthonormal frame fields. Hence, in Sec. II below, we begin with a discussion with such a Galilean transformation.

\section{GALILEAN TRANSFORMATION FOR ROTATIONAL KINEMATIC FORMULA}

Indeed, in the context of general relativity, a rotational Galilean-coordinate transformation under the shared common time coordinate

$$
c t \triangleq \bar{x}^{0} \equiv \check{x}^{0}
$$

from the bar-accented inertial frame to the checkaccented co-rotating frame is given by a space rotation, which is given by

$$
\left\{\begin{array} { l } 
{ \overline { x } ^ { 0 } \equiv \check { x } ^ { 0 } } \\
{ \overline { \mathbf { x } } \equiv \mathbf { R } ( t ; 0 ) \check { \mathbf { x } } }
\end{array} \mapsto \left\{\begin{array}{l}
\check{x}^{0} \\
\check{\mathbf{x}}
\end{array}\right.\right.
$$

with a 3-by-3 orthogonal matrix

$$
\mathbf{R}(t) \triangleq \mathbf{R}(t ; 0) \equiv\left[\mathbf{R}^{\top}(t)\right]^{-1},
$$

with $\mathbf{R}(0) \equiv \mathbf{1}_{\underline{3}}$, that is smooth and continuous over the shared time parameter ' $t$ ' of Eq. (5).

One can trivially verify the matrix for the differentials $\left\{\left\{\mathrm{d} \bar{x}^{\kappa}\right\}\right\} \equiv\left\{\mathrm{d} \bar{x}^{0}, \mathrm{~d} \bar{x}^{1}, \mathrm{~d} \bar{x}^{2}, \mathrm{~d} \bar{x}^{3}\right\}$ that are to be transformed by the rotational Galilean basis transformation;

$$
\left[\begin{array}{l}
\mathrm{d} \bar{x}^{0} \\
\mathrm{~d} \bar{x}^{1} \\
\mathrm{~d} \bar{x}^{2} \\
\mathrm{~d} \bar{x}^{3}
\end{array}\right] \equiv \check{\mathfrak{J}}(\check{\mathfrak{x}})\left[\begin{array}{l}
\mathrm{d} \check{x}^{0} \\
\mathrm{~d} \check{x}^{1} \\
\mathrm{~d} \check{x}^{2} \\
\mathrm{~d} \check{x}^{3}
\end{array}\right]
$$

with a linear transformation matrix of

$$
\check{\mathfrak{J}}(\check{\mathfrak{x}}) \equiv\left(\check{\mathfrak{J}}_{\lambda}^{\kappa}\left(\left\{\left\{\check{x}^{\kappa}\right\}\right\}\right)\right) \triangleq\left(\partial \bar{x}^{\kappa} / \partial \check{x}^{\lambda}\right)_{\check{\Psi}(\wp)},
$$

at coordinate map $\check{\psi}(\wp)$ of point $\wp$, playing the role of the Jacobian matrix.

From now on, as the simplest example of a Galilean transformation of a passive rotation with respect to $\check{x}^{3}$ axis, we consider a simple rotation about the $\bar{x}^{3}=\check{x}^{3}$ axis

$$
\left\{\begin{array} { l } 
{ \overline { x } ^ { 0 } \equiv \check { x } ^ { 0 } } \\
{ \overline { x } ^ { 1 } \equiv \check { x } ^ { 1 } \operatorname { c o s } \varphi _ { \triangleright } ( \check { x } ^ { 0 } ) - \check { x } ^ { 2 } \operatorname { s i n } \varphi _ { \triangleright } ( \check { x } ^ { 0 } ) } \\
{ \overline { x } ^ { 2 } \equiv \check { x } ^ { 1 } \operatorname { s i n } \varphi _ { \triangleright } ( \check { x } ^ { 0 } ) + \check { x } ^ { 2 } \operatorname { c o s } \varphi _ { \triangleright } ( \check { x } ^ { 0 } ) } \\
{ \overline { x } ^ { 3 } = \check { x } ^ { 3 } }
\end{array} \mapsto \left\{\begin{array}{l}
\check{x}^{0} \triangleq c t \\
\check{x}^{1} \\
\check{x}^{2} \\
\check{x}^{3}
\end{array}\right.\right.
$$

for the sake of simplicity for the present discussion by accumulated angle of rotation

$$
\varphi_{\triangleright}\left(\check{x}^{0}\right) \triangleq \int_{0}^{\check{x}^{0} / c} \mathrm{~d} t \cdot \bar{\omega}_{\triangleright}^{3}(c t) .
$$

The differentials of the two coordinates are then transformed based on Eq. (8) rewritten here by a simple relation 
with the 4-by-4 Jacobian matrix

$$
\left(\begin{array}{c}
\mathrm{d} \bar{x}^{0} \\
\mathrm{~d} \overline{\mathbf{x}}
\end{array}\right) \equiv \mathfrak{D}_{(3)}\left(\varphi_{\triangleright}\left(\check{x}^{0}\right) ; \check{\mathbf{x}}\right)\left(\begin{array}{c}
\mathrm{d} \check{x}^{0} \\
\cdots \ldots \\
\mathrm{d} \check{\mathbf{x}}
\end{array}\right)
$$

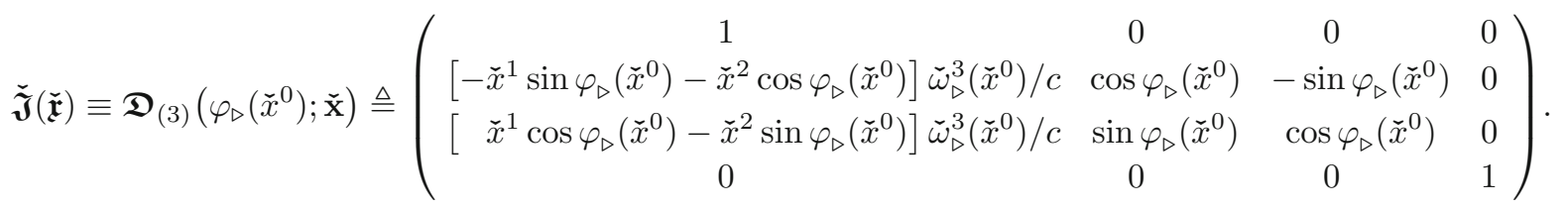

This matrix provides the necessary basis transformation for components of a valid four-vectors across the two frames under the rotatory separation by $\varphi_{\triangleright}\left(\check{x}^{0}\right)$ in Eq. (11) with $t \triangleq \check{x}^{0} / c$, with respect to the common third axis in the Foucault frame depicted in Fig. 1.

Then, the reciprocal matrix to the above $\check{\mathfrak{J}}$ matrix gives a set of four co-frame fields

$$
\begin{aligned}
& \check{\mathfrak{J}}^{-1}(\check{\mathfrak{x}}) \triangleq\left(\partial \check{x}^{\lambda} / \partial \tilde{x}^{\kappa}\right)_{\check{\Psi}(\wp)} \\
& \equiv\left(\begin{array}{cccc}
1 & 0 & 0 & 0 \\
\check{x}^{2} \check{\omega}_{\triangleright}^{3}\left(\check{x}^{0}\right) / c & \cos \varphi_{\triangleright}\left(\check{x}^{0}\right) & \sin \varphi_{\triangleright}\left(\check{x}^{0}\right) & 0 \\
-\check{x}^{1} \check{\omega}_{\triangleright}^{3}\left(\check{x}^{0}\right) / c & -\sin \varphi_{\triangleright}\left(\check{x}^{0}\right) & \cos \varphi_{\triangleright}\left(\check{x}^{0}\right) & 0 \\
0 & 0 & 0 & 1
\end{array}\right) .
\end{aligned}
$$

The familiar relation between the Jacobian matrix and the metric ' $\mathbf{J}^{\top} \mathbf{J}(\mathbf{x}) \equiv \mathbf{g}(\mathbf{x}) \equiv\left(g_{\imath \jmath}\right)$ ' ' in the threedimensional Euclidean manifold is replaced by the one in the Lorentzian manifold,

$$
\begin{gathered}
{[\check{\mathfrak{J}}(\check{\mathfrak{x}})]^{\top}\left(\eta_{\kappa \lambda}\right) \check{\mathfrak{J}}(\check{\mathfrak{x}}) \equiv \check{\mathfrak{g}}(\check{\mathfrak{x}})} \\
\text { for }\left(\eta_{\kappa \lambda}\right) \triangleq \operatorname{diag}\{1,-1,-1,-1\}, \text { i.e. }, \\
\sum_{\mu, \nu=0}^{3} \check{\mathfrak{J}}_{\alpha}^{\mu} \eta_{\mu \nu} \check{\mathfrak{J}}_{\beta}^{\nu} \equiv \check{\mathfrak{g}}_{\alpha \beta}
\end{gathered}
$$

in the four-dimensional space-time. As is the most often the case, summation with each greek-letter index is to be made from zero to three.

Evidently, two expressions for the quadratic form must be equivalent based on the form invariance under the involved transformation;

$$
\begin{aligned}
{[\mathrm{d} s]^{2} \triangleq } & {\left[\mathrm{d} \bar{x}^{0}\right]^{2}-\left[\mathrm{d} \bar{x}^{1}\right]^{2}-\left[\mathrm{d} \bar{x}^{2}\right]^{2}-\left[\mathrm{d} \bar{x}^{3}\right]^{2} } \\
\equiv & {\left[\mathrm{d} \check{x}^{0}\right]^{2}-\left[\mathrm{d} \check{x}^{3}\right]^{2} } \\
& -\left\{\mathrm{d} \check{x}^{1} \cos \varphi_{\triangleright}\left(\check{x}^{0}\right)-\mathrm{d} \check{x}^{2} \sin \varphi_{\triangleright}\left(\check{x}^{0}\right)\right. \\
& \left.-\mathrm{d} \check{x}^{0}\left[\check{x}^{1} \sin \varphi_{\triangleright}\left(\check{x}^{0}\right)+\check{x}^{2} \cos \varphi_{\triangleright}\left(\check{x}^{0}\right)\right] \check{\omega}_{\triangleright}^{3} / c\right\}^{2} \\
& -\left\{\mathrm{d} \check{x}^{1} \sin \varphi_{\triangleright}\left(\check{x}^{0}\right)+\mathrm{d} \check{x}^{2} \cos \varphi_{\triangleright}\left(\check{x}^{0}\right)\right. \\
& \left.+\mathrm{d} \check{x}^{0}\left[\check{x}^{1} \cos \varphi_{\triangleright}\left(\check{x}^{0}\right)-\check{x}^{2} \sin \varphi_{\triangleright}\left(\check{x}^{0}\right)\right] \check{\omega}_{\triangleright}^{3} / c\right\}^{2} \\
\equiv & {\left[\mathrm{d} \check{x}^{0}\right]^{2}-\left[\mathrm{d} \check{x}^{0} \check{\omega}_{\triangleright}^{3}\left(\check{x}^{0}\right) / c\right]^{2}\left\{\left[\check{x}^{1}\right]^{2}+\left[\check{x}^{2}\right]^{2}\right\} } \\
& -\left[\mathrm{d} \check{x}^{1}\right]^{2}-\left[\mathrm{d} \check{x}^{2}\right]^{2}-\left[\mathrm{d} \check{x}^{3}\right]^{2} \\
& +2 \mathrm{~d} \check{x}^{0}\left[\mathrm{~d} \check{x}^{1} \cdot \check{x}^{2}-\check{x}^{1} \mathrm{~d} \check{x}^{2}\right] \check{\omega}_{\triangleright}^{3}\left(\check{x}^{0}\right) / c
\end{aligned}
$$

to be identified by $\sum_{\mu, \nu} \mathrm{d} \check{x}^{\mu} \cdot \check{\mathfrak{g}}_{\mu \nu}\left(\left\{\left\{\check{x}^{\kappa}\right\}\right\}\right) \mathrm{d} \check{x}^{\nu}$. Then, the matrices representing the metric and its reciprocal in the check-accented non-inertial, Foucault frame are found to be

$$
\check{\mathfrak{g}}(\check{\mathfrak{x}}) \equiv\left(\check{\mathfrak{g}}_{\kappa \lambda}\left(\left\{\left\{\check{x}^{\kappa}\right\}\right\}\right)\right) \equiv\left[\begin{array}{cccc}
1-\left\{\left[\check{x}^{1}\right]^{2}+\left[\check{x}^{2}\right]^{2}\right\}\left[\check{\omega}_{\triangleright}^{3}\left(\check{x}^{0}\right) / c\right]^{2} & \check{x}^{2} \check{\omega}_{\triangleright}^{3}\left(\check{x}^{0}\right) / c & -\check{x}^{1} \check{\omega}_{\triangleright}^{3}\left(\check{x}^{0}\right) / c & 0 \\
\check{x}^{2} \check{\omega}_{\triangleright}^{3}\left(\check{x}^{0}\right) / c & -1 & 0 & 0 \\
-\check{x}^{1} \check{\omega}_{\triangleright}^{3}\left(\check{x}^{0}\right) / c & 0 & -1 & 0 \\
0 & 0 & 0 & -1
\end{array}\right]
$$




$$
\check{\mathfrak{g}}^{-1}(\check{\mathfrak{x}}) \triangleq\left(\check{\mathfrak{g}}^{\kappa \lambda}\left(\left\{\left\{\check{x}^{\kappa}\right\}\right\}\right)\right) \equiv\left[\begin{array}{cccc}
1 & \check{x}^{2} \check{\omega}_{\triangleright}^{3}\left(\check{x}^{0}\right) / c & -\check{x}^{1} \check{\omega}_{\triangleright}^{3}\left(\check{x}^{0}\right) / c & 0 \\
\check{x}^{2} \check{\omega}_{\triangleright}^{3}\left(\check{x}^{0}\right) / c & -1+\left[\check{x}^{2}\right]^{2}\left[\check{\omega}_{\triangleright}^{3}\left(\check{x}^{0}\right) / c\right]^{2} & -\check{x}^{1} \check{x}^{2} \check{\omega}_{\triangleright}^{3}\left(\check{x}^{0}\right) / c & 0 \\
-\check{x}^{1} \check{\omega}_{\triangleright}^{3}\left(\check{x}^{0}\right) / c & -\check{x}^{1} \check{x}^{2} \check{\omega}_{\triangleright}^{3}\left(\check{x}^{0}\right) / c & -1+\left[\check{x}^{1}\right]^{2}\left[\check{\omega}_{\triangleright}^{3}\left(\check{x}^{0}\right) / c\right]^{2} & 0 \\
0 & 0 & 0 & -1
\end{array}\right]
$$

along with $\operatorname{det} \check{\mathfrak{g}}(\check{\mathfrak{x}}) \equiv-1$.

The covariant differentials for four-vector velocity $\mathfrak{u}(\tau) \equiv \sum_{\lambda} \check{\mathfrak{u}}^{\lambda}(\tau) \check{\boldsymbol{x}}_{\lambda}$ and four-covector momentum $\mathfrak{p}(\tau) \equiv \sum_{\lambda} \mathbf{d} \check{x}^{\lambda} \cdot \check{\mathfrak{p}}_{\lambda}(\tau)$ are to be written in the transformed non-inertial frame as

$$
\begin{aligned}
& \left\{\begin{array}{l}
\Theta \check{\mathfrak{u}}^{\lambda}(\tau) \\
\widetilde{\mathfrak{p}}_{\lambda}(\tau)
\end{array}\right\} \equiv\left\{\begin{array}{l}
\mathrm{d} \check{\mathfrak{u}}^{\lambda}(\tau) \\
\mathrm{d} \check{\mathfrak{p}}_{\lambda}(\tau)
\end{array}\right\} \\
& +\sum_{\mu, \nu}\left\{\begin{array}{c}
\check{\Gamma}_{\mu \nu}^{\lambda}\left(\left\{\left\{\check{\mathfrak{q}}^{\kappa}(\tau)\right\}\right\}\right) \check{\mathfrak{u}}^{\mu}(\tau) \check{\mathfrak{q}}^{\nu}(\tau) \\
-\check{\mathfrak{p}}_{\mu}(\tau) \check{\Gamma}_{\lambda \nu}^{\mu}\left(\left\{\left\{\check{\mathfrak{q}}^{\kappa}(\tau)\right\}\right\}\right) \check{\mathfrak{q}}^{\nu}(\tau)
\end{array}\right\}, \\
& \left\{\begin{array}{l}
\complement \check{u}^{\lambda}(t) \\
\complement \check{p}_{\lambda}(t)
\end{array}\right\} \equiv\left\{\begin{array}{l}
\mathrm{d} \check{u}^{\lambda}(t) \\
\mathrm{d} \check{p}_{\lambda}(t)
\end{array}\right\} \\
& +\sum_{\mu, \nu}\left\{\begin{array}{c}
\check{\Gamma}_{\mu \nu}^{\lambda}\left(\left\{\left\{\check{q}^{\kappa}(t)\right\}\right\}\right) \check{u}^{\mu}(t) \check{q}^{\nu}(t) \\
-\check{p}_{\mu}(t) \check{\Gamma}_{\lambda \nu}^{\mu}\left(\left\{\left\{\check{q}^{\kappa}(t)\right\}\right\}\right) \check{q}^{\nu}(t)
\end{array}\right\}
\end{aligned}
$$

for $\lambda \in\{0,1,2,3\}$ in terms of the coefficients of the LeviCivita connection [4];

$$
\check{\Gamma}_{\mu \nu}^{\lambda}\left(\left\{\left\{\check{x}^{\kappa}\right\}\right\}\right) \triangleq\left\langle\mathbf{d} \check{x}^{\lambda}, \nabla_{\check{\boldsymbol{x}}_{\nu}} \check{\boldsymbol{x}}_{\mu}\right\rangle
$$

in a torsion-free geometry in a holonomic basis, i.e., coordinate-based frame, which is identified by standard Christoffel symbols [5]

$$
\left\{\begin{array}{c}
\mu \nu \\
\lambda
\end{array}\right\} \triangleq \sum_{\kappa=0}^{3} \check{\mathfrak{g}}^{\lambda \kappa} \frac{\check{\partial}_{\mu} \check{\mathfrak{g}}_{\nu \kappa}+\check{\partial}_{\nu} \check{\mathfrak{g}}_{\mu \kappa}-\check{\partial}_{\kappa} \check{\mathfrak{g}}_{\mu \nu}}{2}
$$

by the so-called Christoffel identity of the second kind.

The number of required summations is not much reduced even in this simple case of a rotation about a single $\bar{x}^{3}=\check{x}^{3}$ axis due to the fact that the basis vectors involved in the above definition is not orthonormal yet. In fact, the nonzero coefficients involved in the frame rotation by the Galilean transformation should be found by $\left\{\left\{\check{\Gamma}_{\mu \nu}^{\lambda}\right\}\right\}$ when either $\mu$ or $\nu$ is or both are zero;

$$
\begin{aligned}
\check{\Gamma}_{\mu 0}^{\lambda}\left(\left\{\left\{\check{x}^{\kappa}\right\}\right\}\right) & \equiv \check{\Gamma}_{0 \mu}^{\lambda} \\
& \equiv \sum_{\kappa} \check{\mathfrak{g}}^{\lambda \kappa} \frac{\check{\partial}_{\mu} \check{\mathfrak{g}}_{\kappa 0}+\check{\partial}_{0} \check{\mathfrak{g}}_{\kappa \mu}-\check{\partial}_{\kappa} \check{\mathfrak{g}}_{\mu 0}}{2}
\end{aligned}
$$

according to Eqs. (22)-(23). In the evaluation of $\left\{\left\{\oplus_{\tau} \check{\mathfrak{u}}^{\lambda}\right\}\right\}$ and $\left\{\left\{\Theta_{t} \check{u}^{\lambda}\right\}\right\}$ as well, we require $\check{\Gamma}_{\mu 0}^{i}$ of $i \in$ $\{1,2,3\}$ and $\mu \in\{0,1,2,3\}$. Their 4-by-4 matrix is found to be

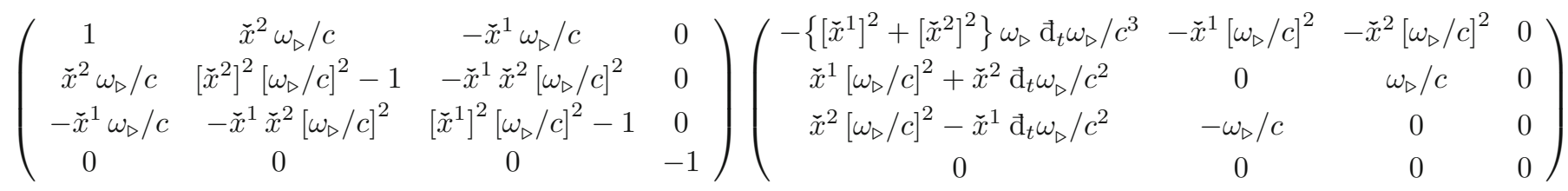

$$
\begin{aligned}
& \equiv\left(\begin{array}{cccc}
0 & 0 & 0 & 0 \\
-\check{x}^{1}\left[\omega_{\triangleright} / c\right]^{2}-\check{x}^{2} \tilde{\mathrm{d}}_{t} \omega_{\triangleright} / c^{2} & 0 & -\omega_{\triangleright} / c & 0 \\
-\check{x}^{2}\left[\omega_{\triangleright} / c\right]^{2}+\check{x}^{1} \mathrm{~d}_{t} \omega_{\triangleright} / c^{2} & \omega_{\triangleright} / c & 0 & 0 \\
0 & 0 & 0 & 0
\end{array}\right)
\end{aligned}
$$

after a substantial simplification for the leading index of the matrix $\lambda=0$, but still with nonzero matrix elements of $\check{\Gamma}_{00}^{1}$ and $\check{\Gamma}_{00}^{2}$.

Note here that the center 2-by-2 block carries the dominant terms in the set of Christoffel symbols that will be responsible for the rotation, whereas the the terms in the first column are responsible for the Galilean transforma- tion representing the rotation. These dominant terms have shown up from $\check{\partial}_{1} \check{\mathfrak{g}}_{20}$ and $\check{\partial}_{2} \check{\mathfrak{g}}_{10}$, which would have been absent in the basis commutation coefficients that will show up in the following analysis with the tetradbased orthonormal frame in the next section. 


\section{TETRAD; ORTHONORMAL FRAME FIELDS FOR ROTATIONAL DYNAMICS}

In order to accommodate the traditional equation of Euler's rotational dynamics, we will consider a socalled tetrad, viz., a set of four orthonormal frame fields $\left\{\left\{\underline{\mathfrak{e}}_{(\kappa)}(\wp)\right\}\right\}$ in the anholonomic orthonormal frame that are rotated from the four basis vectors in the reference inertial frame by the following 4-by-4 matrix

$$
\mathbf{V}^{-1}(\overline{\boldsymbol{\psi}}(\wp)) \triangleq\left(\partial \bar{x}^{\mu} / \partial \xi^{(\alpha)}\right)_{\bar{\psi}(\wp)},
$$

which is constructed by

$$
\mathfrak{O}_{(3)}\left(\varphi_{\triangleright}\left(\bar{x}^{0}\right)\right) \triangleq\left(\begin{array}{cccc}
1 & 0 & 0 & 0 \\
0 & \cos \varphi_{\triangleright}\left(\bar{x}^{0}\right) & -\sin \varphi_{\triangleright}\left(\bar{x}^{0}\right) & 0 \\
0 & \sin \varphi_{\triangleright}\left(\bar{x}^{0}\right) & \cos \varphi_{\triangleright}\left(\bar{x}^{0}\right) & 0 \\
0 & 0 & 0 & 1
\end{array}\right)
$$

on the rotating frame under consideration.

The structure of the above new matrix is simpler than that of ' $\breve{\mathfrak{J}}$ ' in Eq. (13) for the Galilean transformation. The new matrix features the familiar 3 -by-3 subblock that is exactly identical to

$$
\mathbf{O}_{(3)}\left(\varphi_{\triangleright}\right) \triangleq\left(\begin{array}{ccc}
\cos \varphi_{\triangleright} & -\sin \varphi_{\triangleright} & 0 \\
\sin \varphi_{\triangleright} & \cos \varphi_{\triangleright} & 0 \\
0 & 0 & 1
\end{array}\right)
$$

within this limited group of rotations with respect to the $\bar{x}^{3}$ axis warrants the desired effect of the three-vector invariance by the relation

$$
\begin{aligned}
{[\mathrm{d} r]^{2} } & \triangleq\left[\mathrm{d} \bar{q}^{1}(t)\right]^{2}+\left[\mathrm{d} \bar{q}^{2}(t)\right]^{2}+\left[\mathrm{d} \bar{q}^{3}(t)\right]^{2} \\
& \equiv\left[\mathrm{d} \dot{q}^{1}(t)\right]^{2}+\left[\mathrm{d} \dot{q}^{2}(t)\right]^{2}+\left[\mathrm{d} \dot{q}^{3}(t)\right]^{2}
\end{aligned}
$$

under the 3-by-3 matrix ' $\mathbf{O}_{(3)}$ ' above at Eq. (28), whereas $[\mathrm{d} s]^{2}$ in Eq. (17) is now implemented also by

$$
\begin{aligned}
{[\mathrm{d} s]^{2} } & \triangleq\left[\mathrm{d} \overline{\mathfrak{q}}^{0}(\tau)\right]^{2}-\left[\mathrm{d} \overline{\mathfrak{q}}^{1}(\tau)\right]^{2}-\left[\mathrm{d} \overline{\mathfrak{q}}^{2}(\tau)\right]^{2}-\left[\mathrm{d} \overline{\mathfrak{q}}^{3}(\tau)\right]^{2} \\
& \equiv\left[\mathrm{d} \dot{q}^{0}(\tau)\right]^{2}-\left[\mathrm{d} \dot{\mathfrak{q}}^{1}(\tau)\right]^{2}-\left[\mathrm{d} \dot{\mathfrak{q}}^{2}(\tau)\right]^{2}-\left[\mathrm{d} \dot{q}^{3}(\tau)\right]^{2}
\end{aligned}
$$

under the 4 -by-4 matrix ' $\mathcal{O}_{(3)}$ ' above at Eq. (27) in the basis transformation into the special tetrad-based frame with anholonomic, orthonormal basis frame fields. Evidently, the above invariance relation in the tetrad frame is supposed to be valid only locally along the trajectory following the motion of the particle in the four-dimensional manifold that is curved due to the implicit Galilean transformation considered in Sec. II.
1. Vectorial coordinate maps in the orthonormal frame

Remarkably, we can share the above basis transformation, as if it were a regular coordinate transformation for four coordinates of the Galilean transformation. That is, $\stackrel{\mathfrak{q}}{ }^{\alpha}(\tau) \equiv \check{\mathfrak{q}}^{\alpha}(\tau)$ for $\alpha \in\{0,1,2,3\}$, as well as $\stackrel{q}{q}^{0}(t) \equiv \check{q}^{0}(t) \triangleq c t$, and

$$
\check{q}^{i}(t)=\check{q}^{i}(t)
$$

for $i \in\{1,2,3\}$, which renders Eq. (30) into

$$
[\mathrm{d} s]^{2} \equiv[c \mathrm{~d} t]^{2}-\left[\mathrm{d} \dot{q}^{1}(t)\right]^{2}-\left[\mathrm{d} \dot{q}^{2}(t)\right]^{2}-\left[\mathrm{d} \dot{q}^{3}(t)\right]^{2} .
$$

This relation is essentially equivalent to the second line of Eq. (30) at the local point of the particle.

Proper time ' $\tau$ ' of a certain point particle is usually preferred as an absolute scalar for particle dynamics. Yet, in the tetrad-based frame that shares the origin of the original inertial frame, coordinate time ' $t$ ' can also be taken as another scalar parameter from the original inertial frame. That is the special feature in our consideration of the underbar-accented orthonormal tetrad frame for the rotational kinematic formula.

This special feature of the present transformation allows promotion of the space coordinates into a vector that we will refer to the set of components of the vectorial coordinate map in the special manifold de-

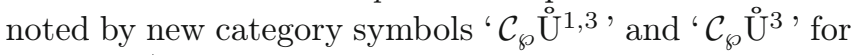
the four-/three-dimensional manifolds of vectorial coordinate maps, respectively, expressed by four/three orthonormal basis vectors. The latter two symbols are distinguished from ' $\mathcal{T}_{\wp} \mathrm{o}^{1,3}$ ' ' and ' $\mathcal{T}_{\wp} \stackrel{\circ}{\mathrm{U}}^{3}$ ' that are reserved for tangent vectors in the respective manifolds.

For such transformation into an anholonomic, orthonormal frame, we now replace the standard coefficients of the metric-based Levi-Civita connection $\check{\Gamma}_{\mu \nu}^{\lambda}\left(\left\{\left\{\check{x}^{\kappa}\right\}\right\}\right)$ of Eq. (22) for a holonomic basis, by the Ricci (connection) coefficients [2]

$$
\underline{\gamma}_{\alpha \beta}^{\lambda}(\wp) \triangleq\left\langle\underline{\mathfrak{e}}^{(\lambda)}, \nabla_{\underline{\mathfrak{e}}_{(\beta)}} \underline{\mathfrak{e}}_{(\alpha)}\right\rangle_{\wp}
$$

over the set of orthonormal, anholonomic basis vectors $\left\{\left\{\mathfrak{e}_{(\kappa)}(\wp)\right\}\right\}$ in Eqs. (26)-(27) and basis covectors, $\left\{\left\{\mathfrak{e}^{(\kappa)}(\wp)\right\}\right\}$, as in

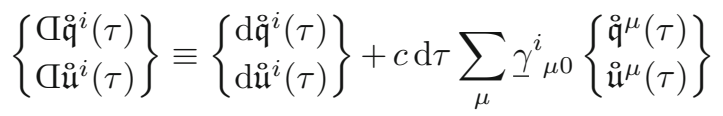

for $i \in\{1,2,3\}$. Derivation of the latter formula is exactly identical to the one in the holonomic case in Eq. (20).

$$
\begin{aligned}
\left\{\begin{array}{c}
\Theta_{t} \boldsymbol{q} \\
\Theta_{t} \boldsymbol{v}
\end{array}\right\} & \equiv \sum_{i=1}^{3} \boldsymbol{r}_{i}(t)\left\{\begin{array}{c}
\Theta_{t} \dot{q}^{i} \\
\Theta_{t} \dot{v}^{i}
\end{array}\right\} \\
& \equiv \sum_{i}\left[\left\{\begin{array}{l}
\mathrm{d}_{t} \dot{q}^{i} \\
\mathrm{~d}_{t} \dot{v}^{i}
\end{array}\right\} \boldsymbol{r}_{i}(t)+\left\{\begin{array}{l}
\dot{q}^{i}(t) \\
\dot{v}^{i}(t)
\end{array}\right\} \Theta_{t} \boldsymbol{r}_{i}\right]
\end{aligned}
$$


paired, not with the holonomic basis covector ' $\mathbf{d} \check{x}^{i}$ ' at Eq. (22), but with the anholonomic basis frame fields

$$
\stackrel{\boldsymbol{r}}{i}_{i}(t) \triangleq \underline{\mathfrak{e}}_{(i)}(\wp)
$$

for $i \in\{1,2,3\}$, derived from the anholonomic basis frame fields $\left\{\left\{\underline{\mathfrak{e}}_{(\ell)}\right\}\right\}$ and the corresponding co-frame fields $\left\{\left\{\underline{\mathfrak{e}}^{(\ell)}\right\}\right\}$ here, to arrive at

$$
\begin{aligned}
\left\{\begin{array}{c}
\Theta_{t} \dot{q}^{i} \\
\Theta_{t} \dot{v}^{i}
\end{array}\right\} & \equiv\left\{\begin{array}{ll}
\left\langle\underline{\mathfrak{e}}^{(i)}(\wp),\right. & \left.\Theta_{t} \boldsymbol{q}\right\rangle \\
\left\langle\underline{\mathfrak{e}}^{(i)}(\wp),\right. & \left.\boldsymbol{G}_{t} \boldsymbol{v}\right\rangle
\end{array}\right\} \\
& \equiv\left\{\begin{array}{c}
\mathrm{d}_{t} \dot{q}^{i} \\
\mathrm{~d}_{t} \dot{v}^{i}
\end{array}\right\}+\sum_{k} \underline{\gamma}_{k 0}^{i}(t) c\left\{\begin{array}{l}
\stackrel{q}{q}^{k}(t) \\
\dot{v}^{k}(t)
\end{array}\right\} .
\end{aligned}
$$

In contrast to greek-lettered summations so far, the summation by each latin letter index is to be made from one to three.

Because ' $t$ ' is another scalar just like the proper time ' $\tau$ ' here, under the Galilean transformation ' $\mathfrak{D}_{(3)}$ ' in Eq. (12) as well as under the present transformation by ' $\mathfrak{O}(3)$ ' in Eq. (27) into the tetrad-based anholonomic frame, we have taken ' $\mathrm{d} t$ ' in the evaluation of the quotient limit, just like taking the quotient limit with $\mathrm{d} \tau$ for Eq. (20). Under a meticulous book-keeping on such vectorial coordinates and covariant velocity vectors, we propose the following two definitions that are taken consecutively;

$$
\begin{aligned}
\left\{\begin{array}{l}
\dot{v}^{i}(t) \\
\dot{\alpha}^{i}(t)
\end{array}\right\} & \triangleq\left\{\begin{array}{l}
\Theta_{t} \dot{q}^{i} \\
\Theta_{t} \dot{v}^{i}
\end{array}\right\} \\
& \equiv\left\{\begin{array}{l}
\mathrm{d}_{t} \dot{q}^{i} \\
\mathrm{~d}_{t} \dot{v}^{i}
\end{array}\right\}+\sum_{j=1}^{3} \underline{\gamma}_{j 0}^{i} \dot{v}^{0}(t)\left\{\begin{array}{l}
\dot{q}^{j}(t) \\
\dot{v}^{j}(t)
\end{array}\right\}
\end{aligned}
$$

for $i \in\{1,2,3\}$ with all the distinctions of derivative symbols carefully made, where we have trivially employed $\stackrel{\circ}{v}^{0}(k) \equiv c$. This is another demonstration of the rotational kinematic formula in Eq. (1). Here, we have exploited the fact that $\gamma_{\alpha 0}^{\lambda} \equiv 0$ when any one of $\{\lambda, \alpha\}$ is zero. This special feature is only observed in this simple case of spatial rotation.

The case of the tetrad for the Lorentz boost, in C. W. Misner et al. [6] in their Eq. (6.6), is the most trivial one, in that the involved basis transformation actually keeps holonomicity as well as symmetry between the tetrad and the co-tetrad. Hence, there is no reason to use either Christoffel symbols or Ricci coefficients. In contrast, the loop-accented tetrad frame that we use in our discussion of rotation is not holonomic, viz., not coordinate-based. The basis of the check-accented Galilean transformation is holonomic, viz., coordinatebased. Yet, the loop-accented, non-inertial frame that one commonly refers to as the "rotating frame" with the "space set of rotated axes" is anholonomic, viz., not coordinate-based, but orthonormal, and will be referred to as a 'tetrad-based frame'.

\section{RICCI CONNECTION COEFFICIENTS FOR AN ANHOLONOMIC BASIS}

Refer to our brief mention on the history of introduction to Ricci connection coefficients in Appendix B. The special relation for the set of basis vectors/covectors in such an anholonomic frame [7],

$$
\begin{aligned}
& \gamma_{\mu \nu}^{\lambda}\left(\left\{\left\{x^{\kappa}\right\}\right\}\right) \\
& \triangleq\left\langle\mathfrak{e}^{\lambda}, \nabla_{\mathfrak{e}_{\nu}} \mathfrak{e}_{\mu}\right\rangle_{(\wp)} \\
& \equiv \sum_{\kappa} \mathfrak{g}^{\lambda \kappa} \frac{\mathfrak{g}_{\nu \kappa, \mu}+\mathfrak{g}_{\mu \kappa, \nu}-\mathfrak{g}_{\mu \nu, \kappa}+c_{\nu \kappa \mid \mu}+c_{\mu \kappa \mid \nu}-c_{\mu \nu \mid \kappa}}{2}
\end{aligned}
$$

after the standard notational convention, ' $\mathfrak{g}_{\nu \kappa, \mu} \equiv$ $\partial_{\mu} \mathfrak{g}_{\nu \kappa}$ ', is the established identity of Ricci connection coefficients in the second-line expression after the firstline definition, while $\left\{\left\{c_{[\mu \nu] \lambda}\right\}\right\} \triangleq\left\{\left\{\sum_{\kappa} c_{[\mu \nu]}{ }^{\kappa} \mathfrak{g}_{\kappa \lambda}\right\}\right\}$ is the set of basis commutation coefficients defined via a Lie derivative [7],

$$
Ł_{\mathfrak{e}_{\mu}} \mathfrak{e}_{\nu} \equiv \nabla_{\mathfrak{e}_{\mu}} \mathfrak{e}_{\nu}-\nabla_{\mathfrak{e}_{\nu}} \mathfrak{e}_{\mu} \triangleq \sum_{\lambda} c_{[\mu \nu]}^{\lambda} \mathfrak{e}_{\lambda}
$$

evidently in a torsion-free geometry in which we use the standard Levi-Civita connection $\left\{\left\{\nabla_{\mathfrak{e}_{(\kappa)}}\right\}\right\}$ among anholonomic basis vectors.

If we support the anholonomic frame by a set of four orthonormal basis frame fields $\left\{\left\{\underline{\mathfrak{e}}_{(\kappa)}\right\}\right\}$ equipped by

$$
\underline{\mathfrak{g}}_{\alpha \beta} \triangleq\left(\underline{\mathfrak{e}}_{(\alpha)} \mid \underline{\mathfrak{e}}_{(\beta)}\right) \triangleq \eta_{\alpha \beta}
$$

for every $\alpha, \beta \in\{0,1,2,3\}$, relation (39) in such a frame is in parallel to the Christoffel identity of the second kind at Eq. (23) [8];

$$
\underline{\gamma}_{\alpha \beta}^{\lambda}\left(\left\{\left\{\check{x}^{\kappa}\right\}\right\}\right) \equiv \sum_{\kappa} \eta^{\lambda \kappa} \frac{\underline{c}_{[\beta \kappa] \alpha}+\underline{c}_{[\alpha \kappa] \beta}-\underline{c}_{[\alpha \beta] \kappa}}{2} .
$$

According to Eq. (40), under the basis transformation into the tefrad frame of Eq. (26), we have

$$
\bigsqcup_{\underline{\mathfrak{e}}_{(0)}} \underline{\mathfrak{e}}(\alpha)_{(\alpha)} \equiv \nabla_{\underline{\mathfrak{e}}_{(0)}} \underline{\mathfrak{e}}(\alpha)_{(\alpha)}-\nabla_{\underline{\mathfrak{e}}_{(\alpha)}} \underline{\mathfrak{e}}(0)_{\beta} \triangleq \sum_{\beta} \underline{c}_{[0 \alpha]}^{\beta} \underline{\mathfrak{e}}_{(\beta)}
$$

with index ' $\alpha$ ' shown in front of ' $\beta$ ' by convention for the standard matrix representation of $\left\{\left\{\underline{c}_{[0 \alpha]} \beta\right\}\right.$. Evidently, for frame-fields in such an orthonormal basis, the two directional derivatives, i.e., the Lie derivative and the covariant derivative, are equivalent. For the frame rotation about the $\check{x}^{3} \equiv \bar{x}^{3}$ axis, the directional derivative gives

$$
\mathfrak{\swarrow}_{\underline{\mathfrak{e}}_{(0)}} \underline{\mathfrak{e}}_{(\alpha)} \equiv \underline{c}_{[0 \alpha]}{ }^{1} \underline{\mathfrak{e}}_{(1)}+\underline{c}_{[0 \alpha]}{ }^{2} \underline{\mathfrak{e}}_{(2)} .
$$

Especially for the present case, one should take a note on $\mathfrak{g}^{i j} \equiv-\delta^{i j}$ for $i, j \in\{1,2,3\}$ under the sign convention 
of the present monograph. Either from physical understanding of the directional derivatives in Eq. (43) with respect to the zeroth frame field or after some elaborate arithmetic workout in the Supplement given below, we find the nonzero center 2-by-2 block

$$
\left(\begin{array}{cc}
\underline{c}_{[01]}^{1} & \underline{c}_{[01]}^{2} \\
\underline{c}_{[02]} & \underline{c}_{[02]}
\end{array}\right) \equiv\left(\begin{array}{cc}
0 & \omega_{\triangleright}(t) / c \\
-\omega_{\triangleright}(t) / c & 0
\end{array}\right)
$$

gives

$$
\begin{aligned}
\left(-\underline{c}_{[0 \alpha] \gamma}\right) & \equiv\left(\underline{c}_{[\alpha 0] \gamma}\right) \triangleq\left(\sum_{\beta} \underline{c}_{[\alpha 0]}^{\beta} \eta_{\beta \gamma}\right) \\
& \equiv\left(\begin{array}{cccc}
0 & 0 & 0 & 0 \\
0 & 0 & \omega_{\triangleright} / c & 0 \\
0 & -\omega_{\triangleright} / c & 0 & 0 \\
0 & 0 & 0 & 0
\end{array}\right)
\end{aligned}
$$

resulting in

$$
\begin{aligned}
\left(\underline{\gamma}_{\beta 0}^{\alpha}\right) & \equiv\left(\sum_{\lambda} \eta^{\alpha \lambda} \frac{\underline{c}_{[\lambda 0] \beta}+\underline{c}_{[\lambda \beta] \sigma}-\underline{c}_{[\beta 0] \lambda}}{2}\right) \\
& \equiv \frac{1}{2}\left(\begin{array}{cccc}
0 & 0 & 0 & 0 \\
0 & 0 & -\omega_{\triangleright} / c-\omega_{\triangleright} / c & 0 \\
0 & \omega_{\triangleright} / c+\omega_{\triangleright} / c & 0 & 0 \\
0 & 0 & 0
\end{array}\right) \\
& \equiv\left(\begin{array}{cccc}
0 & 0 & 0 & 0 \\
0 & 0 & -\omega_{\triangleright} / c & 0 \\
0 & \omega_{\triangleright} / c & 0 & 0 \\
0 & 0 & 0 & 0
\end{array}\right)
\end{aligned}
$$

for Eq. (39) at last. The nonzero structure of this matrix for the Ricci coefficient is particularly simple in contrast to that of the Christoffel symbol in Eq. (25) that is much more complicated. Simplicity of the Ricci coefficient for the tetrad-based orthonormal frame demonstrates advantage of using a tetrad-based frame in comparison to the use of a holonomic Galilean-transformed frame.

\section{Supplement: The standard tetrad formalism from the Galilean frame into the tetrad frame}

Application of the tetrad formalism should be made to the anholonomic locally-inertial rectilinear frame transformed from an arbitrary curved frame. The two frames are respectively denoted by the loop-accent and checkaccent. Henceforth, the resulting tetrad is now given by

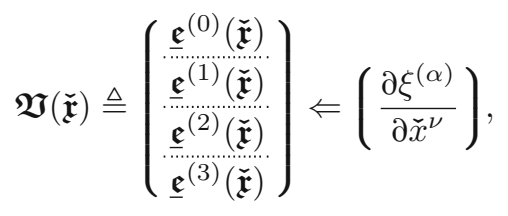

distinguished from ' $\mathbf{V}(\overline{\mathfrak{x}}) \triangleq\left(\partial \xi^{(\alpha)} / \mathrm{\partial} \bar{x}^{\nu}\right)$ ' in Eq. (26) for $\left\{\left\{\xi^{(\alpha)}\right\}\right\}$ being the rectilinear coordinates of the tetrad frame. So, in order to follow this standard usage of the tetrad formalism, we seek such a transformation of $\mathfrak{V}$ with matrix elements

$$
\mathfrak{V}_{\nu}^{(\alpha)}\left(\left\{\left\{\check{x}^{\kappa}\right\}\right\}\right) \triangleq \frac{\partial \xi^{(\alpha)}}{\partial \check{x}^{\nu}} \equiv \sum_{\mu} \frac{\partial \xi^{(\alpha)}}{\partial \bar{x}^{\mu}} \frac{\partial \bar{x}^{\mu}}{\partial \check{x}^{\nu}} .
$$

By use of $\mathfrak{O}_{(3)}^{-1}$ in Eq. (27) and $\check{\mathfrak{J}}(\check{\mathfrak{x}})$ in Eq. (13) for the exemplary rotation about the $\bar{x}^{3} \equiv \check{x}^{3}$ axis,

$$
\mathfrak{V}(\check{\mathfrak{x}}) \equiv \mathfrak{D}_{(3)}^{-1} \cdot \check{\mathfrak{J}}(\check{\mathfrak{x}}) \equiv\left(\begin{array}{cccc}
1 & 0 & 0 & 0 \\
\check{x}^{2} \omega_{\triangleright}(t) / c & 1 & 0 & 0 \\
-\check{x}^{1} \omega_{\triangleright}(t) / c & 0 & 1 & 0 \\
0 & 0 & 0 & 1
\end{array}\right)
$$

for those co-frame fields $\left\{\left\{\underline{\mathfrak{e}}^{(\kappa)}(\check{\mathfrak{x}})\right\}\right\}$ as four row-matrices in the present context within the present supplementary subsection. Their matrix elements in the above expression are expressed all in the check-accented Galilean frame for the set of four co-frame fields of the underbaraccented, anholonomic orthonormal frame. Based on Eq. (50), we find its matrix inverse

$$
\left(\mathfrak{w}_{(\alpha)}^{\mu}\right) \triangleq \mathfrak{V}^{-1}(\check{\mathfrak{x}}) \equiv\left(\begin{array}{cccc}
1 & 0 & 0 & 0 \\
\check{x}^{2} \omega_{\triangleright}(t) / c & 1 & 0 & 0 \\
-\check{x}^{1} \omega_{\triangleright}(t) / c & 0 & 1 & 0 \\
0 & 0 & 0 & 1
\end{array}\right)
$$

With the above two matrices, we may then construct the commutation coefficients by [9]

$$
\underline{c}_{[\alpha \beta]}^{\gamma} \equiv \sum_{\kappa, \lambda}\left[\mathfrak{G}_{(\alpha)}^{\kappa} \check{\partial}_{\kappa} \mathfrak{U}_{(\beta)}^{\lambda}-\mathfrak{U}_{(\beta)}^{\kappa} \check{\partial}_{\kappa} \mathfrak{U}_{(\alpha)}^{\lambda}\right] \mathfrak{V}_{\lambda}^{(\gamma)}
$$

Remarkably, only four coefficients are found to be nonzero as

$$
\begin{aligned}
& \left\{\begin{array}{l}
\underline{c}_{01}{ }^{2} \\
\underline{c}_{02}{ }^{1}
\end{array}\right\} \equiv\left\{\begin{array}{l}
-\underline{c}_{10}{ }^{2} \\
-\underline{c}_{20}{ }^{1}
\end{array}\right\} \\
& \equiv \sum_{\kappa, \lambda}\left\{\begin{array}{l}
{\left[\mathfrak{W}_{(0)}^{\kappa} \check{\partial}_{\kappa} \mathfrak{W}_{(1)}^{\gamma}-\mathfrak{W}_{(1)}^{\kappa} \check{\partial}_{\kappa} \mathfrak{W}_{(0)}^{\lambda}\right] \mathfrak{V}_{\lambda}^{(2)}} \\
{\left[\mathfrak{W}_{(0)}^{\kappa} \check{\check{\partial}}_{\kappa} \mathfrak{W}_{(2)}^{\gamma}-\mathfrak{W}_{(2)}^{\kappa} \check{\partial}_{\kappa} \mathfrak{U}_{(0)}^{\lambda}\right] \mathfrak{V}_{\lambda}^{(1)}}
\end{array}\right\} \\
& \equiv\left\{\begin{array}{lll}
-\mathfrak{U}_{(1)}^{1} & \check{\partial}_{1} \mathfrak{U}_{(0)}^{2} & \mathfrak{V}_{2}^{(2)} \\
-\mathfrak{U}_{(2)}^{2} & \check{\partial}_{2} \mathfrak{U}_{(0)}^{1} & \mathfrak{V}_{1}^{(1)}
\end{array}\right\}
\end{aligned}
$$

after paying attention to the individual terms in the summation. Upon recognizing

$$
\check{\partial}_{1} \mathfrak{U}_{(0)}^{2} \equiv-\omega_{\triangleright} / c \equiv-\check{\partial}_{2} \mathfrak{L}_{(0)}^{1}
$$

from Eq. (51), those nonzero coefficients are evaluated as

$$
\underline{c}_{[01]}^{2}=-\underline{c}_{[02]}^{1} \equiv \omega_{\triangleright} / c .
$$

This result proves Eq. (45), validating the covariant time derivatives in Eq. (37) through the tetrad formalism. 


\section{THE RICCI COEFFICIENT FOR THE ROTATIONAL KINEMATIC FORMULA}

Instead of the above two-step transformation of the four components of the four-vector map in Eq. (50), we now set up a direct relation for the transformation of three components of vectorial maps of different kinds. It is achieved by replacing ' $\mathrm{Cq}_{\mathfrak{q}}{ }^{i}(\tau)$ ' and ' $\mathrm{d} \tau \cdot \mathfrak{q}^{\mu}(\tau)$ ' in Eq. (34) by ' $₫ q^{i}(t)$ ' and ' $\mathrm{d} t \cdot \stackrel{q}{q}^{\mu}(t)$ ', for transformation of the nominal four/three-vector velocity across the two frames with the zeroth coordinate $\xi^{(0)} \equiv c t$ taken at the

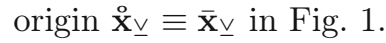

Then, the first row of the matrix relation representing such a transformation in Eqs. (12)-(13) trivially states ' $c \equiv 1 \cdot c$ ', whereas the remaining three rows of Eq. (12) remarkably give

$$
\mathrm{d} \overline{\mathbf{q}} \equiv \mathrm{d} t \cdot \bar{\Omega}_{\triangleright}(t) \overline{\mathbf{q}}(t)+\mathbf{R}(t ; 0) \mathrm{d} \stackrel{\mathbf{q}}{ }
$$

by using matrix symbols for

$$
\overline{\boldsymbol{\Omega}}_{\triangleright}(t) \equiv \boldsymbol{\Xi}_{(3)} \omega_{\triangleright}(t)
$$

with a single matrix of the infinitesimal generator

$$
\boldsymbol{\Xi}_{(3)} \triangleq\left(\begin{array}{ccc}
0 & -1 & 0 \\
1 & 0 & 0 \\
0 & 0 & 0
\end{array}\right)
$$

with the time-ordered product by the Dyson-series solution turning in a rather simple solution

$$
\mathbf{R}(t) \triangleq \mathbf{R}(t ; 0) \equiv \mathbf{O}_{(3)}\left(\varphi_{\triangleright}(t)\right)
$$

at Eq. (28) in the present case.

Generalization of the foregoing rotational Galilean transformation for an arbitrary separation angular velocity $\boldsymbol{\omega}_{\triangleright}(t)$ is now straight-forward. We continue to work with ' $\bar{\Omega}_{\triangleright}\left(t^{\prime}\right)$ ' and ' $\mathbf{R}(t ; 0)$ ', but with the fully generalized expressions for the ever-changing axis and speed of rotation

$$
\overline{\mathbf{\Omega}}_{\triangleright}(t) \equiv \sum_{i=1}^{3} \boldsymbol{\Xi}_{(i)} \bar{\omega}_{\triangleright}^{i}(c t)
$$

which is expressed in terms of $\boldsymbol{\Xi}_{(3)}$ in Eq. (58) and two additional infinitesimal-generators

$$
\boldsymbol{\Xi}_{(1)} \triangleq\left(\begin{array}{ccc}
0 & 0 & 0 \\
0 & 0 & -1 \\
0 & 1 & 0
\end{array}\right), \quad \boldsymbol{\Xi}_{(2)} \triangleq\left(\begin{array}{ccc}
0 & 0 & 1 \\
0 & 0 & 0 \\
-1 & 0 & 0
\end{array}\right)
$$

for a general rotation that is smoothly continuous in time. The set of the latter three matrices constitutes the three infinitesimal generators for matrix Lie algebra $\mathfrak{s o}_{3}(\mathbb{R})$.
We then rewrite Eq. (56) after multiplying $\mathbf{R}^{-1}(t) / \mathrm{d} t$ in front of both sides;

$$
\begin{aligned}
& \stackrel{\mathrm{v}}{ }(t) \triangleq G_{t} \stackrel{\circ}{\mathbf{q}} \\
& \equiv \mathbf{R}^{-1}(t) \overline{\mathrm{d}}_{t} \overline{\mathbf{q}} \\
& =\underbrace{\mathbf{R}^{-1}(t) \overline{\boldsymbol{\Omega}}_{\triangleright}(t) \mathbf{R}}_{\stackrel{\Omega}{\triangleright}_{\triangleright}(t)} \cdot \underbrace{\mathbf{R}^{-1}(t) \overline{\mathbf{q}}(t)}_{\stackrel{\mathrm{q}}{ }(t)}+\underbrace{\mathbf{R}^{-1} \mathbf{R}(t)}_{\mathbf{1}_{\underline{3}}} \cdot \mathrm{d}_{t} \stackrel{\mathbf{q}}{ } \\
& =\stackrel{\circ}{\Omega}_{\triangleright}(t) \cdot \stackrel{\circ}{\mathbf{q}}(t)+\mathbf{1}_{\underline{3}} \cdot \AA_{t} \stackrel{\circ}{\mathbf{q}},
\end{aligned}
$$

where, owing to $\eta^{i j}=-\delta^{i j}$,

$$
\stackrel{\circ}{\triangleright}_{\triangleright}(t) \equiv\left({\stackrel{\circ}{\Omega_{\triangleright j}}}^{\prime}\right) \triangleq\left(c \underline{\gamma}_{\jmath 0}^{\imath}\right) \equiv\left(-c \underline{\gamma}_{\imath \mid \jmath 0}\right)
$$

is apparently the Ricci connection coefficient found in Eq. (47) in the anholonomic frame-field basis, that would play the role of Christoffel symbols for the holonomic basis. This once again verifies the validity of the rotational kinematic formula Eq. (1) in the non-inertial co-rotating frame from the context of a rotational tetrad transformation at Eq. (27) in the language of general relativity.

\section{CONCLUSIONS}

A thorough discussion on the tetrad in the rotating four-dimensional space-time has been made, clarifying many confusing points in great detail. Admittedly, a new interpretation on the rotational kinematic formula requires an elementary knowledge on the curved geometry from the Galilean transformation of the inertial frame into an accelerating frame. Such an elementary knowledge eliminates many, unnecessarily-confusing arguments on two different kinds of time derivatives. The new interpretation of covariant time derivatives based on the tetrad formalism clears out all such confusing explanation of the distinction between the two different kinds. Indeed, one of the most-often encountered textbook examples of a tetrad has been taken with the Schwarzschild geometry around a point-mass gravity, viz., a black hole. The present subject of the rotational kinematic formula should serve as another important example case in learning the subject of tetrad in connection to the Ricci connection coefficients that had shown up quite earlier than the field equation of general relativity.

The author wishes to promote the new interpretation of the rotational kinematic formula that introduces the subject of a tetrad with a subtle distinction between the anholonomic orthonormal frame and the holonomic but non-orthonormal frame made after a Galilean transformation. After all, the new interpretation of the formula is then supposed to eliminate unnecessarily cumbersome, quite varied non-standard notations on the two different kinds of time derivatives. 


\section{ACKNOWLEDGMENTS}

I would like to thank my colleagues, especially Prof. B. S. Mun for encouragement for publication. For technical discussions, special thanks go to Profs. D.-H. Kim and K.-Y. Kim. I would appreciate one of the reviewers greatly, who found an error in my hand simplification of Eq. (25) for the Galilean transformation. The work was supported in part by the GIST Research Institute Fund.

Open Access: This article is licensed under a Creative Commons Attribution 4.0 International License, which permits use, sharing, adaptation, distribution and reproduction in any medium or format, as long as you give appropriate credit to the original author(s) and the source, provide a link to the Creative Commons licence, and indicate if changes were made. To view a copy of this licence, visit http://creativecommons. org/licenses/by/4.0.

\section{APPENDIX A: DIFFERENT SYMBOL CONVENTIONS FOR THE TIME DERIVATIVE OF THE ROTATING OBSERVER}

First of all, the formula in question, Eq. (1) is, not an equation, but an identity. Notably, no good name besides 'kinematic formula' has been attached to this important identity that serves as the foundation of Euler's equations of 1765 for rigid-body dynamics.

- In one of the most authentic classic texts, A. Sommerfeld English ed. [11] in year 1948 had

$$
\left\{\begin{array}{c}
\dot{\mathbf{r}} \\
\dot{\mathbf{v}}
\end{array}\right\}=\frac{\mathrm{d}}{\mathrm{d} t}\left\{\begin{array}{l}
\mathbf{r} \\
\mathbf{v}
\end{array}\right\}+\omega \times\left\{\begin{array}{l}
\mathbf{r} \\
\mathbf{v}
\end{array}\right\}
$$

in his $\S 29$ Eq. (2c) and Eq. (3b).

- Instead of those symbols, one often finds

$$
\left(\frac{\mathrm{d} \mathbf{G}}{\mathrm{d} t}\right)_{\text {space }}=\left(\frac{\mathrm{d} \mathbf{G}}{\mathrm{d} t}\right)_{\text {body }}+\mathbf{\omega} \times \mathbf{G}
$$

as in both H. Goldstein 2nd ed. [12] Eq. (4-120) in year 1980 and H. Goldstein et al. 3rd ed. [13] Eq. (4.81) in year 2002 summarized by an "operator equation"

$$
\left(\frac{\mathrm{d}}{\mathrm{d} t}\right)_{\mathrm{s}}=\left(\frac{\mathrm{d}}{\mathrm{d} t}\right)_{\mathrm{r}}+\omega \times
$$

in his Eq. (4-124) in §4-9 and Eq. (4.82), respectively, in those two editions, with the subscripts referring to "space" and "body (rotating)" systems, respectively.

- In L. D. Landau et al. 3rd ed. [14] Eq. (36.1) in 1976,

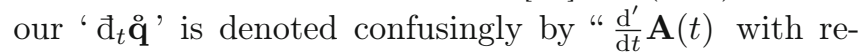
spect to the moving system" distinguished from " $\frac{\mathrm{d}}{\mathrm{d} t} \mathbf{A}(t)$ with respect to the fixed system of co-ordinates";

$$
\frac{\mathrm{d} \mathbf{A}}{\mathrm{d} t}=\frac{\mathrm{d}^{\prime} \mathbf{A}}{\mathrm{d} t}+\boldsymbol{\Omega} \times \mathbf{A},
$$

which has been followed by C. Lanczos 4th ed. [15] Eq. (45.5). In another place Eq. (39.12), L. D. Landau et al. has "velocity

$$
\mathbf{v}_{0}=\mathbf{v}+\Omega \times \mathbf{r}
$$

relative to inertial frame $K_{0}$ ".

- V. I. Arnold 2nd ed. [16] p. 143 in 1989 has

$$
\dot{\mathbf{m}}=B \dot{\mathbf{M}}+[\boldsymbol{\omega}, \mathbf{m}]=B(\dot{\mathbf{M}}+[\mathbf{\Omega}, \mathbf{M}])
$$

with rotating operator " $B_{t}$ " working as " $\mathbf{m}=B_{t} \mathbf{M}(t)$ " with variables of the capital letters expressing "body" variables in the "body frame $K$ ". Indeed, due to different-case letter symbols distinguishing the two frames, the scheme of Arnold's expression of the kinematic formula is at least the most similar to that of our expression in Eq. (1). Arnold uses the above Lie-bracket notation " $[\cdot, \cdot]$ " for the usual vector cross-product.

- For the same relation, as recently as in 2004, T. W. B. Kibble et al. 5th ed. [17] has

$$
\mathrm{d} \mathbf{q} / \mathrm{d} t=\dot{\mathbf{q}}(t)+\boldsymbol{\omega} \wedge \mathbf{q}(t)
$$

in his Eq. (5.7), indeed in the symbol convention opposite to Sommerfeld's use of the dot accent in Eq. (A1). Kibble et al. use the wedge symbol for all the relations requiring the standard cross product.

- R. D. Gregory [18] in 2006 showed two separate formulas; the first one

$$
\left(\frac{\mathrm{d} \boldsymbol{u}}{\mathrm{d} t}\right)_{\mathcal{F}}=\left(\frac{\mathrm{d} \boldsymbol{u}}{\mathrm{d} t}\right)_{\mathcal{F}^{\prime}}+\boldsymbol{\Omega} \times \boldsymbol{u}
$$

for "any vector of time $\boldsymbol{u}$ " in Eq. (17.9) besides the "velocity vector" and the second one for "velocity

$$
\boldsymbol{v}=\boldsymbol{V}+\boldsymbol{\Omega} \times \boldsymbol{r}^{\prime}+\boldsymbol{v}^{\prime}
$$

in his Eq. (17.12) with a trivial augmentation " $\boldsymbol{V}+$ " in the non-relativistic picture, when the origin of the noninertial, rotating frame moves at " $\boldsymbol{V}(t)$ " with respect to the inertial frame. All others including the present monograph do not consider this extra time-varying displacement, because inclusion of the latter is not the point.

- Lastly, M. Spivak [20], p. 333 in year 2010 had

$$
\mathbf{r}^{\prime}=\mathbf{r}^{\prime}+\omega \times \mathbf{r}
$$

and called " $\mathbf{r}$ " "rotating observer's derivative". All these expressions are, in my opinion, quite confusing in comparison to our Eq. (1).

In regard to the extra time-varying displacement represented by the augmented " $\boldsymbol{V}(t)$ " in Eq. (A9) causing extra acceleration, it is evident that the latter extra acceleration should have been dealt with by a so-called Fermi-Walker transport [19] in the tetrad formalism.

In H. Goldstein 2nd ed. [12] §4-1 and §4-9 for kinematics of rigid-body motions, he classified "coordinate axes" either as 
- The "space" set of rotated axes by interpreting time ' $t$ ', as if the set of those axes is observed by an observer at every moment of time or as

- The "body" set of rotating axes by interpreting ' $t$ ' as a variable.

They are used also for two different coordinate systems of time derivatives both for vectors in Cartesian components of the same vector: One is formed under the rotated (space) system for the 'covariant' time differenti-

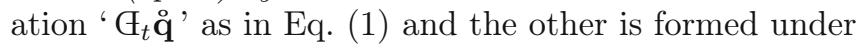
the rotating (body) system for the nominal differentiation ' $\stackrel{\circ}{\mathbf{v}}(t) \triangleq \mathrm{d}_{t} \stackrel{\circ}{\mathbf{q}}$ ' based on the standard formula of Newton's quotient limit.

In the present monograph however, I refrain from using any such confusing terms. As far as I can tell, in all those books, use of covariant symbols, such as ' $G_{t}$ ' in Eq. (35) and ' $\epsilon_{t}$ ' preferred in the present monograph for covariant differentiation, are not found. Contrasting to all those existing practices, based on our recognition of the Ricci connection coefficient, I promote use of new, formal terms such as 'covariant (three-vector) velocity' and 'nominal velocity', which are far more explicit than such terms like velocities in the "space" and the "body" systems, respectively.

\section{APPENDIX B: CHRONOLOGY OF E. B. CHRISTOFFEL, G. RICCI-CURBASTRO, T. LEVI-CIVITA, H. WEYL, AND THEIR CONNECTION COEFFICIENTS}

In the year 1869, Elwin Bruno Christoffel (1829-1900) actually formalized the so-called Riemann tensor, which was expressed by Christoffel symbols [5] " $\left\{\begin{array}{c}i l \\ r\end{array}\right\}$ ". For this reason, the Riemann tensor is often called alternatively the "Riemann-Christoffel tensor". Its tensor symbol with super-/sub-script integer indices, " $\mathcal{R}_{j \mid k l}^{(i)}$ " with every superscript index parenthesized, was devised by Gregorio Ricci-Curbastro (1853-1925), who actually formalized the modern tensor calculus in 1901 [21] with Tulio Levi-Civita (1873-1941).

It seems that the now-standard ' $\Gamma$ '-based symbol for Christoffel's identity in Eq. (22) was chosen firstly by A. Einstein in his paper on November 4, 1915 [22] as " $\Gamma_{\mu \nu}^{\sigma}=-\left\{\begin{array}{c}\mu \nu \\ \sigma\end{array}\right\} "$, conspicuously with the minus sign, and by H. Weyl [23] in 1918 for coefficients of Weyl's affine connection in Eq. (40), even more generalized from Levi-Civita connection in 1917, exactly equivalent to Christoffel symbols of Eq. (23) [4]. Interestingly, Einstein's choice of the capital letter ' $\Gamma$ ' is not quite related to Ricci's choice of the lower-case letter " $\left\{\left\{\gamma_{\imath \mid \jmath \kappa}\right\}\right\}$ " in 1896 [2], which were subsequently named as "coefficients of rotation" in Italian words by Ricci in 1901. However, in 1901, the time coordinate has yet to be included as an additional coordinate of geometry. Anyway, those coefficients are special-case non-tensor symbols for the orthonormal, anholonomic basis [8], analogous to nontensor Christoffel symbols for a holonomic basis generally in a non-orthonormal frame.

In a paper by H. Levy [3], presented to the American Mathematical Society in May 1924 and published in 1925, he had an expression

$$
\left.\frac{\partial \cos \omega_{h i}}{\partial s_{j}}\right|_{P}=\gamma_{h i j},
$$

at p. 143, based on J. Lipka's work of 1924 [24], where " $\omega_{h i}$ is the angle" between two "vectors $\boldsymbol{\lambda}_{h}$ and $\boldsymbol{\mu}_{i}$ at point $P$ on curve $C$, and $s_{j}$ is the arc-length along $C$ ". Unfortunately, Levy's final result above at Eq. (B1) is not quite convenient to use. Instead of the time coordinate explicitly, the two had a certain arc-length parameter. Such a choice appeared to have been made because there was no explicit time coordinate in Ricci's original work in 1896-1900. Also, H. Levy's result should have ' $-\sin [\cdot]$ ' after taking differentiation in Eq. (B1), in contrast to our final formula in Eq. (63).

In 1896, the 'time' coordinate had yet to be recognized as one of the coordinates of the universe. According to H. Levy, Ricci-Curbastro and Levi-Civita themselves in 1901 named $\left\{\left\{\gamma_{\imath \jmath \kappa}\right\}\right\}$ "the coefficients of rotation". Regardless of the original name, I would call them the 'Ricci connection coefficients', for any anholonomic basis, contrasting the term to the 'Christoffel symbols' of the Levi-Civita connection introduced exclusively for any holonomic basis in a torsion-free geometry. Some literatures use " $\left\{\left\{\omega_{\imath \jmath \kappa}\right\}\right\}$ " instead of the original " $\left\{\left\{\gamma_{\imath \jmath \kappa}\right\}\right\}$ ". Ricci-Curbastro [2]. For example in R. M. Wald [25] Eq. (3.4.14), his notation for "Ricci rotation coefficients $\left\{\left\{\omega_{j \kappa \imath}\right\}\right\}$ " follows a different order for the three integer indices.

\section{REFERENCES}

[1] L. Euler, E292 in Euler's Opera Omnia II, 200 (1765), Originally published in Mémoires de l'Académie des Sciences de Berlin 14, 154 (1765).

[2] G. Ricci-Curbastro, Mem. Acc. Lincei. 2, 276 (1896).

[3] H. Levy, Bull. Am. Math. Soc. 31, 142 (1925).

[4] T. Levi-Civita, Rend. Circ. Mat. Palermo 42, 173 (1917).

[5] E. B. Christoffel, J. Reine Angew. Math. 70, 46 (1869), Eqs. (4) and (7).

[6] C. W. Misner, K. S. Thorne, and J. A. Wheeler, Gravitation (W. H. Freeman and Co., New York, USA, 1973). C. W. Misner et al. discusses in general acceleration into the Fermi frame in their Exercise 6.8 in regard to the introduction of the tetrad of the simplest shape by employing " $\Omega^{\mu \nu}$ " in Eq. (6.19) for " $\mathrm{d} \boldsymbol{e}_{\alpha^{\prime}} / \mathrm{d} \tau=-\boldsymbol{\Omega} \cdot \boldsymbol{e}_{\alpha^{\prime}}$ " in their Eq. (6.19). Their " $-\Omega^{\mu \nu}$ " for space indices corresponds to our ' $\Omega_{\triangleright j}^{i}$ ' in Eq. (63) regardless of their choice " $\eta \triangleq \operatorname{diag}\{-1,1,1,1\} "$ for the Minkowski metric. An 
The Rotational Kinematic Formula Viewed from a Tetrad-based Anholonomic Frame - G. Hugh Song

explicit expression for $\left\{\left\{\gamma_{\mu \nu}^{\lambda}\right\}\right\}$ was not given there. Difference between use of ' $\tau$ ' i.e., proper time, and use of $t$ is not an issue.

[7] C. W. Misner et al. op cit., Ref. 6, Eqs. (13.22)-(13.23) and Box 8.4.

[8] L. D. Landau and E. M. Lifshitz, The Classical Theory of Fields, Course of Theoretical Physics, 4th English ed. (Pergamon Press, London, UK, 1975), Eq. (98.11).

[9] P. Szekeres, A Course in Modern Mathematical Physics (Cambridge University Press, London UK, 2004), Prob. 15.26 .

[10] C. W. Misner et al. op cit., [6], Eqs. (9.22)-(9.24).

[11] A. Sommerfeld, Mechanics, English ed. (Academic Press, New York, USA, 1948), §29 Eq. (2c) and Eq. (3b)

[12] H. Goldstein, Classical Mechanics, 2nd ed. (AddisonWesley Pub. Co., Reading, USA, 1980).

[13] H. Goldstein, C. Poole and J. Safko, Classical Mechanics, 3rd ed. (Addison-Wesley Pub. Co., San Francisco, USA, 2002).

[14] L. D. Landau and E. M. Lifshitz, Mechanics, Course of Theoretical Physics (Elsevier Science Ltd, Oxfored, UK, 1976).

[15] C. Lanczos, The Variational Principles of Mechanics, 4th ed. (Dover Publications, Inc., New York, USA, 1970).
[16] V. I. Arnold, Mathematical Methods of Classical Mechanics, 2nd ed. (Springer-Verlag, New York, USA, 1989).

[17] T. W. B. Kibble and F. H. Berkshire, Classical Mechanics, 5th ed. (Imperial College Press, London, UK, 2004).

[18] R. D. Gregory, Classical Mechanics: An Undergraduate Text (Cambridge Universtiy Press, Cambridge, UK, 2006).

[19] C. W. Misner et al. op cit. [6] has $\S 6.5$ on the FermiWalker transport and the final equation in Eq. (6.25) with " $-a$ " for "inertial acceleration".

[20] M. Spivak, Physics for Mathematicians: Mechanics I (Publish or Perish, USA, 2010), p. 333.

[21] M. M. G. Ricci and T. Levi-Civita, Math. Ann. B 54, 125 (1901).

[22] A. Einstein, Königlich Preußische Akademie der Wissenschaften (Berlin). Sitzungsberichte (Nov. 4, 1915), Eq. (15). It is the paper with a mistaken field equation " $R_{\mu \nu}=-\varkappa T_{\mu \nu}$ " published one month earlier than the milestone Dec. 2 paper of Einstein.

[23] H. Weyl, Math. Z. 2, 384 (1918), pf. 390.

[24] J. Lipka, "On Ricci's coefficients of rotation", J. Math. Phys. 3, 7 (1924).

[25] R. M. Wald, General Relativity (The University of Chicago Press, Chicago, USA, 1984), Eq. (3.4.14). 